\title{
European Concerted Action on Anticoagulation. A multicentre calibration study of WHO international reference preparations for thromboplastin, rabbit (RBT/90) and human (rTF/95)
}

\author{
L Poller, M Keown, N Chauhan, A M H P van den Besselaar, A Tripodi, C Shiach, J Jespersen
}

J Clin Pathol 2005;58:667-669. doi: 10.1136/icp.2004.019810

A 10 centre calibration was performed after six years to determine the international sensitivity index (ISI) of rTF/95 relative to RBT/90, and to assess any international normalised ratio (INR) bias compared with the original multicentre calibration. After exclusion of one outlying centre, the follow up calibration gave a mean ISI for rTF/95 of 0.99 , which although a small difference, is significantly greater than the mean ISI of 0.94 obtained previously. The change in ISI for international reference preparation (IRP) rTF/95 relative to RBT/90 would lead to a slight bias in INR for human compared with rabbit thromboplastins. At a theoretical INR of 3.0 , the INR bias is $6.0 \%$, and this is below the accepted $10 \%$ level of clinical relevance. Ongoing stability monitoring of World Health Organisation thromboplastin IRP is advised.

$\mathrm{T}$ he World Health Organisation (WHO) prothrombin time (PT) standardisation scheme derives from a mathematical determination of the responsiveness of individual PT test systems. The manual PT results with a thromboplastin international reference preparation (IRP) are compared with the local PT system using orthogonal regression. ${ }^{1}$ International sensitivity index (ISI) calibrations need to be species specific using the three different IRPs-that is, human (rTF/95, ISI 0.94), rabbit (RBT/90, ISI 1.0), and bovine (OBT/79, ISI 1.0).

"The WHO prothrombin time standardisation scheme derives from a mathematical determination of the responsiveness of individual prothrombin time test systems"

The rabbit and human preparations originally calibrated in $1991^{2}$ and $1995,{ }^{3}$ respectively, are mainly used. No provision was made for their longterm stability monitoring. In a European Concerted Action on Anticoagulation (ECAA) study in 2001 reported by Poller et $a l,{ }^{4}$ both these IRPs were calibrated at 10 centres. The relation between the ISI of the IRP with results obtained in 1995, reported by Tripodi et al, ${ }^{3}$ is examined in our present study to determine whether it has remained constant.

\section{MATERIALS AND METHODS}

Blood was drawn into $105 \mathrm{mmol} /$ litre sodium citrate (Vacutainer; Becton Dickinson, Oxford, UK), centrifuged, and plasma transferred into plastic tubes; the tubes were capped at room temperature and the blood was tested within six hours.
The 10 ECAA centres calibrating rTF/95 used fresh citrated plasma from 20 normal controls and 60 patients according to the WHO protocol. Plasma from each subject was tested in parallel with rTF/95 and RBT/90. ${ }^{1}$ Plasma samples were tested with the two IRPs in a fixed sequence provided to all centres. ${ }^{5}$ All donors gave informed consent.

\section{STATISTICAL ANALYSIS}

The ISI for rTF/95 was derived against RBT/90, and the imprecision of the calibration line slope was expressed as a coefficient of variation (CV(b)), according to WHO guidelines. ${ }^{1}$ Only the assumption that the mean log PT of normal controls lies on the calibration line derived from patients' PT values was assessed. ${ }^{6}$

Between centre ISI variation was measured using CV (\%). Outlying ISIs were detected by means of an algorithm..$^{23}$

A two sample $t$ test was performed to compare the ISI of rTF/95 in the ECAA study with the mean ISI of the Tripodi et al study. ${ }^{3}$ Between centre variations in the ISI for the two studies were compared ( $F$ test).

The prothrombin ratio (PR) for rTF/95, corresponding to a theoretical international normalised ratio (INR) of 3.0, was calculated with the mean ISI from the Tripodi et al calibration. ${ }^{3}$ This PR was then used to calculate an INR with the mean ISI obtained in our present study, and the absolute percentage deviation from a theoretical INR of 3.0 was determined. Absolute INR deviation exceeding 10\% was deemed clinically relevant. ${ }^{4}$

\section{RESULTS}

Tables 1 and 2 show the individual centre results for the 1995 Tripodi et al study, ${ }^{3}$ and for the later ECAA study, respectively. In the later study, one centre gave an outlying ISI. After exclusion of this centre, the ECAA gave a mean ISI of 0.99 , which is significantly different from the mean ISI of 0.94 obtained by Tripodi and colleagues ${ }^{3}$ (95\% confidence interval for difference in mean ISI, 0.01 to 0.09 ; two sample $t$ test, $\mathrm{p}=0.009$ ).

The $\mathrm{CV}(\mathrm{b})$ of the calibration slope for the ECAA study ranged from $1.9 \%$ to $4.5 \%$ (mean $\mathrm{CV}(\mathrm{b}), 3.0 \%$ ). Six centres reported a $\mathrm{CV}(\mathrm{b})$ within the $3 \%$ limit set by the WHO guidelines. ${ }^{1}$ For the Tripodi et al study, ${ }^{3}$ the $\mathrm{CV}(\mathrm{b})$ ranged from $1.8 \%$ to $4.0 \%$ (mean $\mathrm{CV}(\mathrm{b}), 2.8 \%$ ). Thirteen centres reported a $\mathrm{CV}(\mathrm{b})$ below $3 \%$.

Abbreviations: CV, coefficient of variation; ECAA, European Concerted Action on Anticoagulation; INR, international normalised ratio; IRP, international reference preparation; ISI, international sensitivity index; PR, prothrombin ratio; PT, prothrombin time; WHO, World Health Organisation 
Table 1 Multicentre ISI calibration of rTF/95 against RBT/90 reported by Tripodi and colleagues ${ }^{3}$

\begin{tabular}{|c|c|c|c|c|c|c|}
\hline Centre & $\mathbf{N}$ & ISI & $\mathrm{CV}(\mathrm{b})$ & Slope of patients only line & INR (RBT/90) & INR (rTF/95) \\
\hline 1 & 73 & 0.85 & 2.8 & 0.80 & 2.94 & 3.22 \\
\hline 2 & 72 & 0.97 & 4.0 & 1.06 & 2.75 & 2.67 \\
\hline 3 & 78 & 0.99 & 1.8 & 1.12 & 2.79 & 2.70 \\
\hline 4 & 75 & 0.94 & 3.3 & 0.87 & 2.78 & 2.77 \\
\hline 5 & 78 & 0.95 & 2.4 & 0.94 & 2.59 & 2.54 \\
\hline 6 & 79 & 0.96 & 2.1 & 0.99 & 2.74 & 2.71 \\
\hline 7 & 69 & 0.92 & 3.3 & 1.02 & 2.66 & 2.77 \\
\hline 8 & 80 & 0.88 & 2.8 & 0.92 & 2.43 & 2.58 \\
\hline 9 & 80 & 0.94 & 3.4 & 0.95 & 2.72 & 2.74 \\
\hline 10 & 72 & 0.93 & 2.2 & 0.95 & 2.86 & 2.83 \\
\hline 11 & 67 & 1.05 & 3.5 & 1.04 & 2.56 & 2.31 \\
\hline 12 & 78 & 0.90 & 2.7 & 0.94 & 2.62 & 2.79 \\
\hline 13 & 79 & 0.94 & 2.7 & 0.92 & 2.43 & 2.44 \\
\hline 14 & 73 & 0.98 & 2.0 & 1.03 & 2.75 & 2.71 \\
\hline 15 & 75 & 0.95 & 2.3 & 1.02 & 2.87 & 2.89 \\
\hline 16 & 74 & 0.92 & 3.5 & 0.93 & 2.41 & 2.45 \\
\hline 17 & 68 & 0.96 & 2.2 & 0.92 & 2.90 & 2.77 \\
\hline 18 & 74 & 0.87 & 2.8 & 0.93 & 2.40 & 2.58 \\
\hline 19 & 79 & 0.99 & 2.5 & 1.08 & 2.62 & 2.53 \\
\hline Overall & & 0.94 & 2.8 & 0.97 & 2.67 & 2.68 \\
\hline CV (\%) & & 5.0 & & 7.9 & & \\
\hline
\end{tabular}

No calibration resulted in significant displacement of the mean log normal PT from the patients only line.

Tables 1 and 2 show the mean INR of the patients' samples calculated with the established ISI values for rTF/95 and RBT/ 90 .

Between centre ISI differences were slightly greater in our present calibration (ISI range, 0.80-1.05; CV, 7.0\%) than in the first by Tripodi and colleagues ${ }^{3}$ (ISI range, 0.85-1.05; CV, $5.0 \%$ ). Between centre variations in the ISI for the two calibrations were not significantly different ( $F$ test, $p=0.2$ ).

A PR of 3.2 gave a theoretical INR of 3.0 with the mean ISI of 0.94 in the original Tripodi et al report. ${ }^{3}$ After exclusion of the outlying centre, this PR gave an INR of 3.2 with the mean ISI of 0.99 from the ECAA study $(6.0 \%$ difference from a theoretical INR of 3.0).

\section{DISCUSSION}

It is reassuring that no gross change has occurred in the ISI relation between the two main WHO reference thromboplastins over the six year interval between the two calibrations.

A small change in the ISI for rTF/95 relative to RBT/90, similar to the change seen in our present calibration was noted in a report in 2002, ${ }^{8}$ but was not significant. The trend to a slightly greater ISI for rTF/95 relative to RBT/90 reported here would lead to a small INR bias between thromboplastins calibrated with these IRPs. However, the INR bias at INR 3.0 is $6 \%$, which is not greater than the accepted $10 \%$ level of clinical relevance.

The average INR of the patients in the first study was slightly greater than in the second, but there was no significant correlation between local ISI and mean INR. This suggests that any differences in ISI were not caused by different mean patient INR values, and is in agreement with the mean log PT of normals lying on the line derived only from the patients' PT.

"It is reassuring that no gross change has occurred in the international sensitivity index relation between the two main WHO reference thromboplastins"

Although there were differences in centres, technicians, patient and normal samples, and collection tubes between the two multicentre calibrations, a change in the physical

Table 2 Multicentre ISI calibration of rTF/95 against RBT/90 performed by the ECAA in 2001

\begin{tabular}{|c|c|c|c|c|c|c|}
\hline Centre & $\mathrm{N}$ & ISI & $C V(b)$ & Slope of patients only line & INR (RBT/90) & INR (rTF/95) \\
\hline$A$ & 75 & 0.97 & 3.1 & 0.95 & 2.41 & 2.33 \\
\hline$B$ & 73 & 0.99 & 2.2 & 1.08 & 3.09 & 2.95 \\
\hline C & 70 & 1.02 & 2.7 & 1.17 & 2.90 & 2.73 \\
\hline D & 76 & 0.99 & 3.2 & 0.97 & 2.44 & 2.35 \\
\hline $\mathrm{E}$ & 73 & 0.95 & 1.9 & 1.00 & 2.96 & 2.96 \\
\hline $\mathrm{F}$ & 78 & $0.80^{*}$ & 4.5 & 0.81 & 2.20 & 2.51 \\
\hline G & 75 & 0.97 & 2.3 & 1.06 & 2.24 & 2.21 \\
\hline $\mathrm{H}$ & 73 & 1.05 & 2.7 & 1.14 & 2.58 & 2.37 \\
\hline I & 71 & 1.03 & 2.8 & 0.92 & 2.87 & 2.56 \\
\hline j & 74 & 0.96 & 4.5 & 0.96 & 2.16 & 2.14 \\
\hline Overall & & 0.97 & 3.0 & 1.00 & 2.58 & 2.51 \\
\hline Between laboratory CV & & 7.0 & & 10.9 & & \\
\hline Overall, excluding F & & 0.99 & 2.8 & 1.03 & 2.63 & 2.51 \\
\hline Between laboratory CV & & 3.5 & & 8.6 & & \\
\hline
\end{tabular}

$\mathrm{N}$ is the total number of samples used in each calibration. $\mathrm{CV}(\mathrm{b})$ is the coefficient of variation of the calibration slope for healthy subjects plus patients. The mean INR for the patients, samples included in the calibration was calculated from RBT/90 and rTF/95 measurements.

* Detected as an outlying result.

CV, coefficient of variation; ECAA, European Concerted Action on Anticoagulation; INR, international normalised ratio; ISI, international sensitivity index. 


\section{Take home messages}

- We carried out a 10 centre calibration to determine the international sensitivity index (ISI) of rTF/95 relative to RBT/90, and to assess any international normalised ratio (INR) bias compared with the original multicentre calibration

- There was a small change in the ISI for rTF/95, which would lead to a slight bias in INR for human compared with rabbit thromboplastins, although at a theoretical INR of 3.0, this bias is below the accepted $10 \%$ level of clinical relevance

- Ongoing stability monitoring of World Health Organisation thromboplastin international reference preparations is recommended

or chemical properties of $\mathrm{rTF} / 95$ or $\mathrm{RBT} / 90$ cannot be excluded as a cause of the difference between the two calibrations.

RBT/90 will be replaced soon and both rTF/95 and RBT/90 (and OBT/79) should be included in the proposed calibration. ${ }^{9}$ Any INR bias between the two routes of calibration would thus be minimised.

The CVs of the slopes at all centres were acceptable, all being less than 5\%. However, the 3\% CV limit may be too ambitious for a cross species calibration, as shown by the fact that four centres in the later study exceeded this figure.

An ongoing programme of stability monitoring is recommended for thromboplastin IRPs. This could be similar to the 10 year study of the British Committee of Standards for Haematology for the WHO human thromboplastin IRP, BCT/ 253. ${ }^{10}$ Alternatively, multicentre ISI calibrations should be repeated at four years intervals.

\section{ACKNOWLEDGEMENTS}

Additional multicentre study participants: J Conard, Laboratoire Central D’Hématologie, Hôtel-Dieu de Paris, Paris, France; D Dias, Service Immunotherapia, Hospital de S Joao, 4760 Vila Nova de Famalicao, Porto, Portugal; N Egberg, Department of Clinical Chemistry, Karolinska Hospital, Stockholm, Sweden; JA Iriarte, Instituto de Epidemiologia y Prevencion de Enfermedades Cardiovasculares, Hospital Civil de Basurto, Bilbao, Spain; I Kontopoulou-Griva, Anticoagulant Unit, Hippocration General Hospital, Athens, Greece; B Otridge, Haematology Department, Mater Misericordiae Hospital, Dublin, Ireland.

We are also grateful to the following scientific staff for their valuable assistance: G Anthi (Athens, Greece), M Clerici (Milan, Italy), H Fitzgerald (Dublin, Ireland), MH Horellou (Paris, France), J Meeuwisse-Braun (Leiden, the Netherlands), EM Norberg and
L Söderblom (Stockholm, Sweden), K Overgaard (Esbjerg, Denmark), M Vacas Rius (Bilbao, Spain).

\section{Authors' affiliations}

L Poller, M Keown, N Chauhan, ECAA Central Facility, Faculty of Life Sciences, University of Manchester, Manchester M13 9PT, UK

A M H P van den Besselaar, Haemostasis and Thrombosis Research Centre, Leiden University Medical Centre, 2300 RC Leiden, The Netherlands

A Tripodi, A Bianchi Bonomi, Haemophilia and Thrombosis Centre, IRCCS Maggiore Hospital, University of Milan, 20122 Milan, Italy C Shiach, Department of Haematology, Manchester Royal Infirmary, Oxford Road, Manchester M13 9WL, UK

J Jespersen, Department for Thrombosis Research, University of Southern Denmark and Department of Clinical Biochemistry, Ribe County Hospital, Esbjerg, DK-6700 Denmark

The study was supported by EC Standards, Measurements and Testing Programme (grant number SMT4-CT98-2269) and an additional research grant from the Manchester Thrombosis Research Foundation. Gratitude is expressed to WHO Biologicals for the supply of RBT/90 and rTF/95 IRP.

Correspondence to: Professor L Poller, ECAA Central Facility, Faculty of Life Sciences, The University of Manchester, Manchester; ecaa@ manchester.ac.uk

Accepted for publication 29 October 2004

\section{REFERENCES}

1 WHO Expert Committee on Biological Standardisation. Guidelines for thromboplastins and plasma to control oral anticoagulant therapy. World Health Organ Tech Rep Ser 1999;889:64-93.

2 Van den Besselaar AMHP. Multicenter study of replacement of the international reference preparation for thromboplastin, rabbit, plain. Thromb Haemost 1993;70:794-9.

3 Tripodi A, Chantarangkul V, Negri B, et al. International collaborative study for the calibration of a proposed reference preparation for thromboplastin, human recombinant, plain. Thromb Haemost 1998;79:439-43.

4 Poller L, Keown M, Chauhan N, et al. Multicentre international sensitivity index calibration of two types of point-of-care prothrombin time monitor systems. Br J Haematol 2002;1 16:844-50

5 Poller L, Keown M, Chauhan N, et al. Comparison of fresh plasma and whole blood multicentre ISI calibrations of CoaguChek Mini and TAS PT-NC whole blood prothrombin time point-of-care monitors. Thromb Haemost 2002;87:859-66.

6 Tomenson JA. A statistician's independent evaluation. In: Van den Besselaar AMHP, Lewis SM, Gralnick HR, eds. Thromboplastin calibration and oral anticoagulant control. Boston: Martinus Nijhoff Publishers, 1984:87-108.

7 Poller L, van den Besselaar AMHP, Jespersen J, et al. The effect of sample size on fresh plasma thromboplastin ISI determination. Br J Haematol 1999; 105:655-63.

8 Van den Besselaar AMHP, Tripodi A. A reassessment of the relationship between international reference preparations for human and rabbit thromboplastins. Thromb Haemost 2002;88:459-61.

9 Tripodi A, Poller L, van den Besselaar AMHP, et al. A proposed scheme for calibration of international reference preparations of thromboplastin for the prothrombin time. Thromb Haemost 1995;74:1368-9.

10 Poller L, Pulford J, Stevenson KJ, et al. Long term stability studies on the WHO IRP thromboplastin (human plain BCT/253). Thromb Haemost 1994;72:682-4. 